\title{
SHM via topological derivative
}

\author{
A Martínez ${ }^{1}$, J A Güemes ${ }^{2}$, J M Perales ${ }^{3}$ (i) and J M Vega ${ }^{1}$ (1) \\ ${ }^{1}$ Department of Applied Mathematics, Universidad Politécnica de Madrid, UPM, Plaza Cardenal Cisneros \\ 3, E-28040-Madrid, Spain \\ ${ }^{2}$ Center of Composites Materials and Smart Structures, Universidad Politécnica de Madrid, UPM, Plaza \\ Cardenal Cisneros 3, E-28040-Madrid, Spain \\ ${ }^{3}$ Department of Aircraft and Spacecraft, Universidad Politécnica de Madrid, UPM, Plaza Cardenal \\ Cisneros 3, E-28040-Madrid, Spain \\ E-mail: josemanuel.vega@upm.es
}

Received 22 March 2018, revised 10 May 2018

Accepted for publication 24 May 2018

Published 4 July 2018

\begin{abstract}
This paper deals with the use of the topological derivative as an analysis tool for structural health monitoring, to locate the presence of flaws in a homogeneous material plate that is subject to guided elastic waves excitation. Using a numerical solver to compute the response of the system and defining a scalar objective function that measures the least squares difference between the measured and calculated signals, the topological derivative somehow describes the sensitivity of the objective function to localized perturbations of the material properties due to the presence of defects. Thus, defects are guessed to be located near the topological derivative peaks. This is somehow related to the minimization of the objective function and uses the whole physics of the problem (to compute the objective function), instead of a smaller amount of physical information, as conventional methods do. Here, we reconstruct small defects via the topological derivative by using multi-frequency synthetic data, for several representative configurations of the actuators and sensors, and several defect locations. Among these, some fairly demanding configurations are considered that are not accessible to conventional methods, such as actuators and sensors located very close to the plate boundary, and defects located beyond both elongated through-slits and elongated inclusions of a different material.
\end{abstract}

Keywords: non-destructive testing, defect detection, elastic waves, multi-frequency, topological derivative, inverse problems

(Some figures may appear in colour only in the online journal)

\section{Introduction}

Structural health monitoring (SHM) using forced guided waves [1] has attracted considerable attention in the literature in recent years, specially to monitor structural plates. In addition to nondestructive testing of structures [2], similar problems arise in a number of fairly different fields, such as medical diagnosis [3], natural resources exploration [4], oceanography [5], and location of anti-personnel landmines [6].

Generation and sensing of guided waves can be performed by various means, either piezoelectric [7, 8] or noncontact optical laser [9-13] actuators and sensors. However, non-contact generation of these waves is somewhat problematic from the practical point of view, either using optical laser methods [14] or even some more recent magnetostrictive techniques [15], because such non-contact generation requires high-power devices.
Mathematically, processing data from elastic waves in a material medium to identify defects and inclusions (which affect material parameters such as the Young modulus, the Poisson ratio, and the density) is an inverse problem [16]. Inverse problems consist in guessing the properties of a physical system from its response to excitation. These problems have been formulated and treated in mathematics for years and give rise to formulations that can be set in stimulating/provocative terms $[17,18]$. The name inverse problem comes as opposite to the direct problem, which consists in calculating the system response when its physical properties are known. It is convenient to note that inverse problems are usually ill-posed from the mathematical point of view [19].

There is a variety of mathematical methods to solve inverse problems [20]. An appealing approach is to formulate the problem as an optimization problem, in which the objective function accounts for the difference between the experimentally 

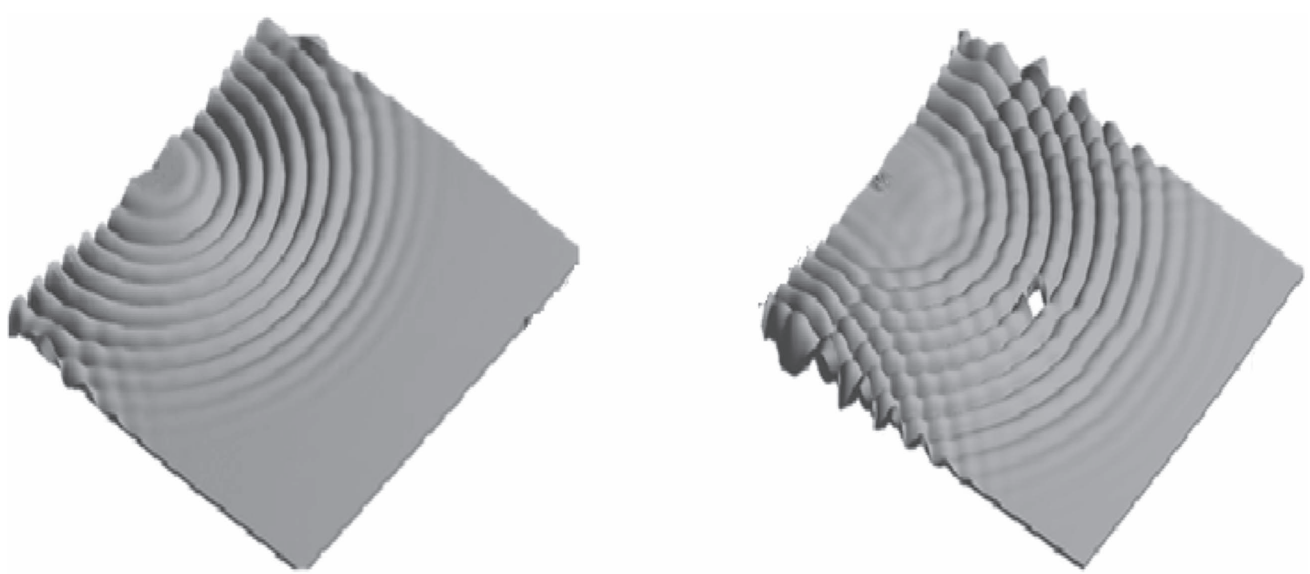

Figure 1. Sketch of propagative Lamb waves produced from a localized actuator in a healthy (left) and damaged (right) plate, showing reflection at the boundary of the plate and scattering at the defect. The damaged plate contains the indicated through-hole defect.

measured and computed (for varying values of the material properties) responses of the system. The guessed values of the material properties are those that minimize the objective function. Thus, in the context of SHM, this formulation could give the defects location and intensity. For the simple case of a through-hole defect in a plate, with guided waves produced in a localized source, figure 1 illustrates how scattering at the defect affects the waves and produces differences from their counterparts in the healthy plate. In the simplest conventional methods, the emitted waves (from may be various actuators to shape the excitation) are pulses that are received at various sensors. The localization of the defects is computed using the time-lags between the emitted and received pulses, and the wave propagation velocity, which is known. By their own nature, these methods are very flexible because they only use the propagation velocity and, thus, are transparent to the wave motion physics behind, e.g., elastic waves, electromagnetic waves, water waves, et cetera. However, they also exhibit strong limitations due to the mixing of the primary emitted waves and their reflection at the lateral walls, which is visible in figure 1 and may lead to very complex wave patterns [21, 22]. Also, the good functioning of these methods require appropriate location of the sensors [23]. These limitations manifest themselves in cases in which:

- The actuators and/or sensors are close to the boundaries, which strongly mixes the primary and reflected waves near the actuators and/or sensors, thus widening the width of the received signals.

- The defects are beyond elongated through-slits, elongated inclusions of a different material, or attached elongated structural strips (such as stringers in an aircraft wing), which necessarily diffract/scatter the primary waves before these reach the defects and sensors.

These difficulties are due to the fact that conventional methods use a limited part of the involved physics only. The most primitive conventional methods only use the propagation velocity, which allows for computing the 'time of flight', but ignores diffraction. There are some, more elaborate conventional methods that do take diffraction into account using variants of tomographic inspection [24-28]. The present approach in terms of an optimization problem, instead, does use the whole physics (which includes reflection, diffraction, and scattering) in the computation of the objective function. In other words, the optimization problem approach uses much more detailed information about the physics and should, in principle, provide a more precise solution than conventional methods. In fact, as it will be seen along the paper, the proposed approach solves the difficulties mentioned above in connection with conventional methods. In particular, in the present approach: (i) both the actuators and sensors will be very close to the boundary, and (ii) the method will be seen to be able to detect defects that are beyond elongated through-slits and other elongated artifacts that would mask defects in conventional methods.

The above mentioned optimization problem can be treated by iterative methods [29]. However, these methods either require a good initial condition (which, in turn, necessitate a prior knowledge of the number of defects) or involve a high number of iterations. A promising alternative to plain optimization methods is based on the computation of the so-called topological derivative [30-32], which has been already used in the contexts of acoustic waves [33] and elastic waves [34-38] and somehow gives the sensitivity of the objective function to local perturbations (from healthy conditions) in the material properties. The idea is that localized defects are expected to be located in those regions where the sensitivity is largest, namely near the topological derivative peaks. The topological derivative can be seen as the gradient of the objective function, and may be computed by solving an appropriate adjoint problem. This method can be applied to any actuators/sensors configuration and to any emitted signal.

Obviously, the larger the number of (uncorrelated) available data the better the obtained results. Here, we consider emitted signals consisting in combinations of monochromatic excitations and conveniently merge the topological derivatives associated with the individual monochromatic excitations for various actuators/sensors configurations. The 

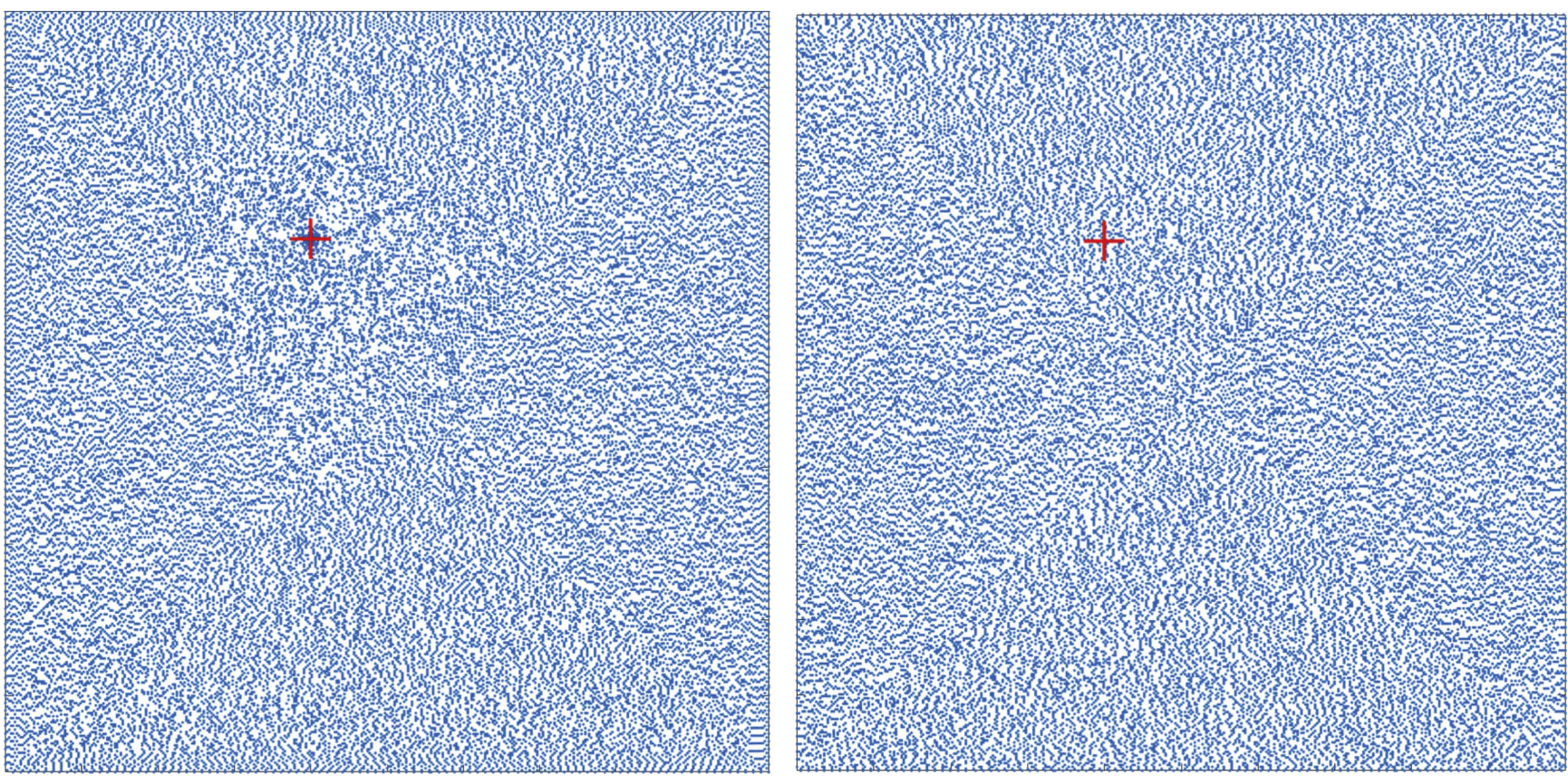

Figure 2. The meshes used to compute the synthetic data (left) and to solve the direct and adjoint problems (right) in the computations considered in figure 3-left, showing not the whole amount of nodes (which would make too dense plots), but only one out each of 4 nodes. The defect location is indicated with a red + symbol.

performance of the method will be tested considering various representative defect locations. This approach has been already pursued in the (somewhat academic) context of the two-dimensional wave equation, with very good results [39]. The idea is extended in the present paper to a much more realistic elastic plate problem, intended as a proof of the concept in the SHM context. To this end, the whole approach will be simplified in various ways, which will also enhance clarity in the presentation:

- Instead of actual experimental data, numerically computed synthetic data will be used. Experimental noise will be mimicked by using fairly different numerical grids in the synthetic data computations and the calculation of the topological derivative; see figure 2. Actual experimental noise could be treated using higher order dynamic mode decomposition (HODMD), a very recent method [40] that also de-convolutes multi-frequency data. HODMD has proved to be very effective in treating experimental data resulting from particle image velocimetry measurements [41, 42], laser imaging detection and ranging measurements [43], and aircraft flight tests [44]. However, the combination of topological derivative and HODMD is beyond the scope of this paper.

- Only symmetric Lamb waves [45] will be considered, as resulting from symmetric in-plane excitation. As anticipated, this excitation may be produced by various contact and non-contact actuators, symmetrically located at both sides of the plate. This simplification will allow for a twodimensional approximation that both reduces the computational cost of the computations (which will be made using ANSYS Mechanical [46]) and clarifies presentation of the results.
- Consistently with the two-dimensional (plane stress) approximation, the defects will be assumed to be (twodimensional) through-holes trespassing the plate. More general non-trespassing, symmetrically located defects with various widths break the plane stress assumption, but their effect in the present model can be guessed to be roughly proportional to the defect volume. For instance, a symmetric defect with a thickness equal to $50 \%$ of the plate thickness will roughly have the same effect as a half in-plane area through-hole defect. However, a consistent analysis of these more general defects would require using a full three-dimensional model, whose combination with the methodology in this paper is straightforward, except for the much larger computational cost.

- Sensors will mimic (synthetically) either piezoelectric sensors or laser Doppler vibrometers.

- Although a large number of cases have been considered and analyzed, for the sake of brevity only the most representative ones will be presented. In particular, all results below will deal with transducers located very close to the boundary and very small defects, which are the most demanding cases. For transducers not so close to the boundary and/or larger defects, the defect detection is much better than in the cases presented below.

With the above in mind, the remainder of the paper is organized as follows. The considered elastic problem and its two-dimensional approximation are set in section 2 , where the definition and computation of the topological derivative are also described. The main results of the paper are given and discussed in section 3 and some concluding remarks, in section 4 . 


\section{Formulation and topological derivative}

The elastodynamic problem for symmetric Lamb waves in a plate is first formulated in section 2.1, where a two-dimensional approximation is used taking advantage of the fact the plate thickness is small compared to the transversal plate dimensions. Then, an appropriate objective function is defined in section 2.2, where the computation of the associated topological derivative is also described.

\subsection{Formulation of the elastic problem}

We consider a rectangular metallic plate of size $\left(2 L_{1}\right) \times$ $\left(2 L_{2}\right) \times d$. As anticipated in section 1 , we shall only consider symmetric Lamb waves [45]. Assuming that the waves wavelength is large compared to the plate thickness $d$ and that this thickness is small compared to both $2 L_{1}$ and $2 L_{2}$, the elastodynamic state admits a plane stress assumption, which allows for a two-dimensional approximation. Using a Cartesian coordinate system with the origin at the center of the plate and the $x$ and $y$ axes parallel to the plate sides, the plate is the rectangle $D$ : $-L_{1}<x<L_{1},-L_{2}<y<L_{2}$. With the usual notation, the in-plane displacement vector $\vec{u}=(u, v)$, assumed to be conveniently small, obeys the following linear elastodynamics equation

$\rho \frac{\partial^{2} \vec{u}}{\partial t^{2}}-\nabla(\lambda \nabla \cdot \vec{u})-\nabla \cdot\left[\mu\left(\nabla \vec{u}+\nabla \vec{u}^{\top}\right)\right]=\vec{f}(x, y, t)$

in the domain $D$, with boundary conditions

$$
\begin{gathered}
\lambda(\nabla \cdot \vec{u}) \vec{n}+\mu\left(\nabla \vec{u}+\nabla \vec{u}^{\top}\right) \cdot \vec{n}=\overrightarrow{0} \quad \text { at } \\
x= \pm L_{1},|y|<L_{2} \text { and } y= \pm L_{2},|x|<L_{1}, \\
\vec{u}=\overrightarrow{0} \quad \text { at }(x, y)=\left( \pm L_{1},-L_{2}\right) .
\end{gathered}
$$

These impose pure reflection at the lateral walls, in (2), and eliminate rigid-body displacements, in (3). In this formulation, $\rho$ is the density, $t$ denotes time, $\lambda$ and $\mu$ are the Lamé coefficients, ${ }^{\top}$ denotes the transpose, the forcing term $\vec{f}$ depends on the actuators configuration, and $\vec{n}$ is the outward unit normal to the boundary of $D$. Note that equations (1) and (2) can also be written as $\rho \partial^{2} \vec{u} / \partial t^{2}-\nabla \cdot \overline{\bar{\sigma}}=\vec{f}$ and $\overline{\bar{\sigma}} \cdot \vec{n}=\overrightarrow{0}$, respectively, where the stress tensor $\overline{\bar{\sigma}}$ is defined as

$$
\overline{\bar{\sigma}}=\lambda(\nabla \cdot \vec{u}) \overline{\bar{I}}_{2}+2 \mu \overline{\bar{\varepsilon}} .
$$

Here, $\overline{\bar{I}}_{2}$ is the $2 \times 2$ unit tensor and $\overline{\bar{\varepsilon}} \equiv\left(\nabla \vec{u}+\nabla \vec{u}^{\top}\right) / 2$ is the strain tensor, whose components are given by

$$
\begin{aligned}
\varepsilon_{11} & =\frac{\partial u}{\partial x}, \quad \varepsilon_{12}=\varepsilon_{21}=\frac{1}{2}\left[\frac{\partial u}{\partial y}+\frac{\partial v}{\partial x}\right], \\
\varepsilon_{22} & =\frac{\partial v}{\partial y}
\end{aligned}
$$

Since the solution to the problem (1)-(3) is linear on the forcing term $\vec{f}$ (though not on the material properties $\rho, \lambda$, and $\mu$ !), for multi-frequency excitation produced by $M$ forcing devices, of the form

$$
\vec{f}(x, y, t)=\sum_{k=1}^{K} \sum_{m=1}^{M} \vec{F}_{k m}(x, y) \cos \left(\omega_{k} t+\phi_{k}\right),
$$

the dynamics of the displacement vector can be set into the form

$$
\vec{u}(x, y, t)=\sum_{k=1}^{K} \sum_{m=1}^{M} \vec{U}_{k m}(x, y) \cos \left(\omega_{k} t+\phi_{k}\right) .
$$

Substituting (6)-(7) into (1)-(3) and setting to zero the various coefficients of $\cos \left(\omega_{k} t+\phi_{k}\right)$ yield the following purely spatial problems (for $k=1, \ldots, K$ )

$$
\begin{aligned}
& \nabla\left(\lambda \nabla \cdot \vec{U}_{k m}\right)+\nabla \cdot\left[\mu\left(\nabla \vec{U}_{k m}+\nabla \vec{U}_{k m}^{\top}\right)\right] \\
& \quad+\rho \omega_{k}^{2} \vec{U}_{k m}=-\vec{F}_{k m}(x, y),
\end{aligned}
$$

in $D$, with boundary conditions

$$
\begin{gathered}
\lambda\left(\nabla \cdot \vec{U}_{k m}\right) \vec{n}+\mu\left(\nabla \vec{U}_{k m}+\nabla \vec{U}_{k m}^{\top}\right) \cdot \vec{n}=\overrightarrow{0} \text { at } \\
x= \pm L_{1},|y|<L_{2} \text { and } y= \pm L_{2},|x|<L_{1}, \\
\vec{U}_{k m}=\overrightarrow{0} \quad \text { at }(x, y)=\left( \pm L_{1},-L_{2}\right) .
\end{gathered}
$$

Now, if the forcing terms $\vec{F}_{k m}$ appearing in (6) are all real, equations (8)-(10) show that the displacement fields $\vec{U}_{k m}$ are also real, which means that each monochromatic component in the expansion (7) represents a standing wave. However, depending on the frequencies $\omega_{k}$ and the phases $\phi_{k}$, the whole spatio-temporal pattern (7) may also be a standing wave or a propagative wave. For instance, by applying Fourier transform, the propagative pulses considered in conventional methods can be expanded in the form (6)-(7). In the sequel, all computations will be based on the purely spatial problems (8)-(10), which means that the phases $\phi_{k}$ appearing in (6)-(7) will play no role. This makes a significant difference with conventional methods, in which the phases do play an important role. This strategy based on (8)-(10) will be followed for the sake of simplicity in the presentation, though a more general approach that takes the phases into account could improve the obtained results.

As anticipated in section 1, the aim of the present paper is to identify defects in the plate by appropriate post-process of the data obtained in some sensors when the plate is subject to appropriate forcing. Defects will manifest themselves in the values of the material properties of the plate, namely the density and the Lamé coefficients, which are assumed to be of the form

$$
\rho=\rho_{0}-\widetilde{\rho}, \quad \lambda=\lambda_{0}-\widetilde{\lambda}, \quad \mu=\mu_{0}-\widetilde{\mu},
$$

where the subscript ${ }_{0}$ denotes the values of the material properties for the healthy plate and the perturbations, denoted with tildes, are due to the presence of localized defects. These are assumed to be appropriately small in the $L_{2}$ norm, namely 
to be such that

$$
\begin{gathered}
\int_{D}|\tilde{\rho}|^{2} d x d y \ll \int_{D} \rho_{0}^{2} d x d y, \quad \int_{D}|\tilde{\lambda}|^{2} d x d y \\
\ll \int_{D} \lambda_{0}^{2} d x d y, \int_{D}|\tilde{\mu}|^{2} d x d y \ll \int_{D} \mu_{0}^{2} d x d y .
\end{gathered}
$$

In the present context, these conditions will hold because of the localization of the defects, in spite of the fact that the perturbations in the coefficient values need not be small in the defects themselves. In this case, equation (12) just means that the area occupied by the defects must be small compared to the area of the plate. For instance, circular defects with a diameter equal to one tenth of the plate sides, exhibit an area that is one hundred of that of the plate.

Concerning the in-plane forcing terms $\vec{F}_{k m}$ appearing in (8), we shall consider a set of $M$ simple forcing configurations, each (for $m=1, \ldots, M$ ) of one of the two types:

- Point localized (PL) excitation, in which a concentrated force at a given point $\left(x_{m}, y_{m}\right)$ is imposed. Thus, the forcing term appearing in (8) is given by

$$
\vec{F}_{k m}^{\mathrm{PL}}(x, y)=\vec{a}_{k m} \delta\left(x-x_{m}, y-y_{m}\right),
$$

where the vector $\vec{a}_{k m}$ is the applied force and $\delta$ is the Dirac delta function. This excitation mimics the noncontact optical laser actuators.

- Rotationally symmetric radial (RSR) excitation, in which a RSR force is applied in a circle with center at a given point $\left(x_{m}, y_{m}\right)$ and radius $r$. Thus, the forcing term appearing in (8) is given by

$$
\vec{F}_{k m}^{\mathrm{RSR}}(x, y)=a_{k m} \vec{g}\left(x-x_{m}, y-y_{m}, r\right),
$$

where the scalar $a_{k m}$ is the overall intensity of the applied force and $\vec{g}\left(x-x_{m}, y-y_{m}, r\right)$ denotes a forcing term consisting in applying a unit force along the outward normals to the circle of radius $r$, centered at $\left(x_{m}, y_{m}\right)$. This mimics piezoelectric actuators.

Likewise, the resulting synthetic data, mimicking experimental data, will be collected in $N$ sensors (for $n=1$, $\ldots, N$ ), each of one of the following two types (which are the counterparts of the two types of excitation considered above):

- Point localized (PL) sensing, in which the displacement vector at a given point $\left(x_{n}, y_{n}\right)$ is measured. This sensing device mimics laser Doppler sensors and produces two scalar measurements (namely, the two components of the displacement vector) at the sensor.

- Rotationally symmetric radial (RSR) sensing in a circle with center at a given point $\left(x_{n}, y_{n}\right)$ and radius $r$, measuring the spatial average of the radial displacement vector along the circle. This mimics piezoelectric sensors of radius $r$ centered at $\left(x_{n}, y_{n}\right)$ and produces just one scalar measurement, the averaged radial displacement vector.

The forcing and sensing types (either PL or RSR, as described above) will affect the computation of the topological derivative in the next subsection.
According to our comments above, these excitation/sensing types will be combined in section 3 in three ways, namely PLforcing/PL sensing, RSR-forcing/RSR sensing, and RSR-forcing/PL sensing, considering $M$ actuator configurations, which will be common to the $K$ considered frequencies. For each of these configurations, appropriate data will be collected at $N$ sensors, all of them of the same type (either PL or RSR). Thus, the available scalar data is $K \times M \times(2 N)$ for PL sensing and $K \times M \times N$ for RSR sensing. However, the unknowns in the inverse problem are the spatial distributions of the perturbations $\tilde{\rho}, \tilde{\lambda}$, and $\tilde{\mu}$, which involve an infinite number of scalar unknowns from the purely mathematical point of view. Upon discretization via a spatial mesh, with $P$ nodes, the number of unknowns is $3 P$, which is usually much larger than the number of available uncorrelated data. This is one of the difficulties involved in inverse problems, which are generally ill-posed. An additional, even more important, difficulty is that the unknown material properties do not depend continuously on the given data.

\subsection{Objective function and topological derivative}

As already explained in section 1, our approach will consist in somehow minimizing a scalar objective function, which accounts for the least squares difference between the measured data at the sensors and their computed counterpart for a given varying location and shape of the defects. The defects manifest themselves in the present context through the distributions of the perturbations $\widetilde{\rho}, \widetilde{\lambda}$, and $\widetilde{\mu}$, as defined in (11). In principle, minimizing the objective function defined that way should provide an approximation of $\widetilde{\rho}, \widetilde{\lambda}$, and $\widetilde{\mu}$, and thus the location and shape of the defects when these are localized.

The above mentioned objective function is defined as the sum of the contributions from the $K$ forcing frequencies and the $M$ actuators/sensors configurations, namely

$$
\begin{aligned}
& \mathcal{H}(\widetilde{\rho}, \widetilde{\lambda}, \widetilde{\mu})=\sum_{k=1}^{K} \sum_{m=1}^{M} \alpha_{k m} \mathcal{H}_{k m}^{\mathrm{PL}} \text { and } \\
& \mathcal{H}(\widetilde{\rho}, \widetilde{\lambda}, \widetilde{\mu})=\sum_{k=1}^{K} \sum_{m=1}^{M} \alpha_{k m} \mathcal{H}_{k m}^{\mathrm{RSR}},
\end{aligned}
$$

for PL and RSR sensing, respectively, which must be treated separately. Here, as above, $\widetilde{\rho}, \widetilde{\lambda}$, and $\widetilde{\mu}$ denote the perturbations (from the healthy state, according to (11)) in these properties appearing in the damaged plate, the subscripts $k$ and $m$ label the frequencies and actuators/sensors configurations, respectively, the weights $\alpha_{k m}>0$ (to be selected below) measure the individual contribution of each frequency and each emitting configuration, and $\mathcal{H}_{k m}^{\mathrm{PL}}$ and $\mathcal{H}_{k m}^{\mathrm{RSR}}$ depend on the sensing mode.

For PL sensing,

$$
\begin{aligned}
\mathcal{H}_{k m}^{\mathrm{PL}}(\widetilde{\rho}, \widetilde{\lambda}, \widetilde{\mu})= & \frac{1}{2} \sum_{n=1}^{N} \| \vec{U}_{k m}^{\text {comp. }}\left(x_{m n}, y_{m n}\right) \\
& -\vec{U}_{k m}^{\text {meas. }}\left(x_{m n}, y_{m n}\right) \|^{2},
\end{aligned}
$$

where $\left(x_{m n}, y_{m n}\right)$ denote the sensor positions (which may depend on the actuators set configuration), $\vec{U}_{k m}$ is the $k$ th Fourier component of the displacement vector field produced 
by the $m$ th excitation, as defined in (7), and the superscripts comp. and meas. denote computed (by solving (8)-(10)) and measured data. In the present case, we are using synthetic data, mimicking actual measured data, which means that $U^{\text {meas. }}$ will also be computed by solving (8)-(10) for the actual damaged plate. Since (either actual or synthetic) measurements are known beforehand, dependence of $\mathcal{H}_{k m}^{\mathrm{PL}}$ on $\widetilde{\rho}, \widetilde{\lambda}$, and $\widetilde{\mu}$ comes from $\vec{U}_{k m}^{\text {comp. }}$, whose calculation requires solving (8)-(10), which in turn depend on $\widetilde{\rho}, \widetilde{\lambda}$, and $\widetilde{\mu}$. In other words, $U^{\text {meas. }}$ will be computed by solving (8)-(10) for the known distributions of $\widetilde{\rho}, \widetilde{\lambda}$, and $\widetilde{\mu}, U^{\text {comp. }}$ will be computed by solving (8)-(10) for varying distributions of while $\widetilde{\rho}, \widetilde{\lambda}$, and $\widetilde{\mu}$, which should be selected to minimize the objective function.

Similarly, for RSR sensing,

$$
\mathcal{H}_{k m}^{\mathrm{RSR}}(\widetilde{\rho}, \widetilde{\lambda}, \widetilde{\mu})=\frac{1}{2} \sum_{n=1}^{N}\left\langle\vec{U}_{k m}^{\text {comp. }}-\vec{U}_{k m}^{\text {meas. }}\right\rangle_{x_{m n}, y_{m n}, r}^{2},
$$

where $\vec{U}_{k m}^{\text {comp. }}$ and $\vec{U}_{k m}^{\text {meas. }}$ are as above and for a vector field $\vec{U},\langle\vec{U}\rangle_{x_{m n}, y_{m n}, r}$ denotes the average of the outward normal component of $\vec{U}$ on the circle of radius $r$ centered at the point $\left(x_{m n}, y_{m n}\right)$. Note that $\langle\vec{U}\rangle_{x_{m n}, y_{m n}, r}$ is a scalar quantity in spite of the fact that $\vec{U}$ is a vector field. Again, dependence of $\mathcal{H}_{k m}^{\mathrm{RSR}}$ on $\widetilde{\rho}, \widetilde{\lambda}$, and $\widetilde{\mu}$ comes through $\vec{U}_{k m}^{\text {comp. }}$.

As anticipated in section 1, minimizing the objective function (15) should give a good guess of the unknowns $\widetilde{\rho}, \widetilde{\lambda}$, and $\widetilde{\mu}$. However, the objective function depends nonlinearly on the unknowns and this approach is problematic. Thus, it is substituted in this paper by a guess based on a more direct, noniterative process that uses the topological derivative of the objective function $\mathcal{H}$, which is a scalar vector field $\mathcal{T}(x, y)$ defined at each $(x, y) \in D$ as follows. We consider a small circular defect, with center at $(x, y)$ and radius $r$. The topological derivative is the limit

$$
\mathcal{T}(x, y)=\lim _{r \rightarrow 0} \frac{\mathcal{H}^{r}-\mathcal{H}^{0}}{\pi r^{2}},
$$

where $\mathcal{H}^{r}$ and $\mathcal{H}^{0}$ denote the values of the objective function for the damaged and healthy plates, respectively. In other words, the topological derivative at $(x, y)$ measures the sensitivity of the objective function to an infinitesimal defect located at this point. The idea is that damage is most likely to occur at those points of the plate where the topological derivative exhibits negative peaks. Note that the defects considered in (18) are infinitesimal, which means that considering them as circular is only a slight restriction and the actual defects that are to be localized may have an arbitrary shape. They will be just located at those regions where the function $\mathcal{T}$ exhibits the most pronounced negative values, which will be easily identified from convenient color maps of the topological $\mathcal{T}$.

The topological derivative is a linear operator, which means invoking (15) that

$$
\mathcal{T}(x, y)=\sum_{k=1}^{K} \sum_{m=1}^{M} \alpha_{k m} \mathcal{T}_{k m}(x, y),
$$

where $\mathcal{T}_{k m}$ is the topological derivative of $\mathcal{H}_{k m}^{\mathrm{PL}}$ or $\mathcal{H}_{k m}^{\mathrm{RSR}}$ for PL sensing or RSR sensing, respectively, defined in (16) and
(17), respectively. Using well-known computations in the literature [47], it follows that

$$
\mathcal{T}_{k m}=\rho \mathcal{S}_{k m}^{\rho}+\frac{1}{\mu(1+\nu)}\left[\frac{\nu-1-2 \nu^{2}}{2\left(1-\nu^{2}\right)} \mathcal{S}_{k m}^{\lambda}+\mathcal{S}_{k m}^{\mu}\right],
$$

where $\nu=\lambda /[2(\lambda+\mu)]$ is the Poisson ratio and

$$
\begin{aligned}
& \mathcal{S}_{k m}^{\rho}=-\omega_{k}^{2} \vec{U}_{k m}^{d} \cdot \vec{U}_{k m}^{a}, \\
& \mathcal{S}_{k m}^{\lambda}=\left(\nabla \cdot \vec{U}_{k m}^{d}\right)\left(\nabla \cdot \vec{U}_{k m}^{a}\right), \\
& \mathcal{S}_{k m}^{\mu}=\frac{1}{2}\left(\nabla \vec{U}_{k m}^{d}+\nabla \vec{U}_{k m}^{d \top}\right): \\
&\left(\nabla \vec{U}_{k m}^{a}+\nabla \vec{U}_{k m}^{a \top}\right) .
\end{aligned}
$$

Here, $\vec{U}_{k m}^{d}$ and $\vec{U}_{k m}^{a}$ are the solutions of the unperturbed direct and adjoint problems, which are both given by (8)-(10) with $\rho=\rho_{0}, \lambda=\lambda_{0}, \mu=\mu_{0}$ (the unperturbed values of these properties for the healthy plate) and the following forcing term in the right hand side of (8), $\vec{F}_{k m}$ :

- For the unperturbed direct problem, $\vec{F}_{k m}$ depends on the forcing type and is given by (13) and (14) for PL and RSR-forcing, respectively.

- For the unperturbed adjoint problem, $\vec{F}_{k m}$ depends on the sensing type, either PL or RSR. For PL sensing at the points $\left(x_{m n}, y_{m n}\right)$, for $n=1, \ldots, N$, we have

$$
\begin{aligned}
\vec{F}_{k m} & =\sum_{n=1}^{N}\left[\vec{U}_{k m}^{d}\left(x_{m n}, y_{m n}\right)-\vec{U}_{k m}^{\text {meas. }}\left(x_{m n}, y_{m n}\right)\right] \\
& \times \delta\left(x-x_{m n}, y-y_{m n}\right),
\end{aligned}
$$

where $\delta$ is the Dirac delta function, as above. For RSR sensing in devices of radius $r$, centered at the points $\left(x_{m n}\right.$, $\left.y_{m n}\right)$, for $n=1, \ldots, N, \vec{F}_{k m}$ is given by

$$
\begin{aligned}
\vec{F}_{k m} & =\sum_{n=1}^{N}\left\langle\vec{U}_{k m}^{d}-\vec{U}_{k m}^{\text {meas. }}\right\rangle_{x_{m n}, y_{m}, r}, \\
& \times \vec{g}\left(x-x_{m n}, y-y_{m n}, r\right),
\end{aligned}
$$

where the rotationally symmetric vector field $\vec{g}$ and the mean value $\langle\cdot\rangle_{x_{m n}, y_{m n}, r}$ are as defined after (14) and (17), respectively. Recall that the azimuthal average $\langle\cdot\rangle_{x_{m n}, y_{m n}, r}$ is a scalar quantity.

Once the unperturbed direct and adjoint problems have been solved, equations (19)-(22) allow to compute the topological derivative field $\mathcal{T}(x, y)$, which still depends on the weights $\alpha_{k m}$. Following ideas in [39], which gave very good results for the two-dimensional wave equation, we select

$$
\alpha_{k m}=\frac{1}{\left|\min _{(x, y) \in D_{I}} \mathcal{T}_{k m}(x, y)\right|}
$$

where $D_{I} \subset D$ is an interrogation window, which is the subset of the plate where the topological derivative will be calculated. Such interrogation window must exclude a vicinity of the actuators and sensors because the topological derivative takes very large (positive or negative) values in near these devices. 
This is because the solutions to the unperturbed direct and adjoint problems are very steep near the actuators and sensors, respectively, and the topological derivative includes terms (namely, the second and third terms in the right hand side of equation (20)) that are proportional to the product of the gradients of the unperturbed direct and adjoint problems. The large values of the topological derivative near the actuators/sensors could give negative peaks that would be spurious in the present context and must be avoided.

It is interesting to note that the fields $\mathcal{S}_{k m}^{\rho}, \mathcal{S}_{k m}^{\lambda}$, and $\mathcal{S}_{k m}^{\mu}$, defined in (21)-(22) admit another interpretation, as sensitivities of $\mathcal{H}_{k m}$ to perturbations in $\rho, \lambda$, and $\mu$, respectively. In other words, if $\rho, \lambda$, and $\mu$ are perturbed as in (11), with conveniently small perturbations, according to (12), the individual components of the objective function defined in (16) can be linearized around $\tilde{\rho}=\tilde{\lambda}=\tilde{\mu}=0$, which (using a standard continuous adjoint formulation [48]) gives

$$
\begin{aligned}
\mathcal{H}_{k m}(\widetilde{\rho}, \tilde{\lambda}, \widetilde{\mu})-\mathcal{H}_{k m}^{0} \simeq \int_{D}\left[\mathcal{S}_{k m}^{\rho}(x, y) \tilde{\rho}(x, y)\right. \\
\left.+\mathcal{S}_{k m}^{\lambda}(x, y) \tilde{\lambda}(x, y)+\mathcal{S}_{k m}^{\mu}(x, y) \tilde{\mu}(x, y)\right] d x d y,
\end{aligned}
$$

where $\mathcal{H}_{k m}^{0}$ is the unperturbed value of $\mathcal{H}_{k m}$, quadratic and higher order terms in the small quantities $\tilde{\rho}, \tilde{\lambda}$, and $\tilde{\mu}$ have been neglected, and the sensitivities $\mathcal{S}_{k m}^{\rho}, \mathcal{S}_{k m}^{\lambda}$, and $\mathcal{S}_{k m}^{\mu}$ are precisely those given by (21)-(22), with the unperturbed direct and adjoint problems as defined above. Thus, equation (20) represents a particular linear combination of these sensitivities. Other linear combinations could give better results, as could do a selection of the weights $\alpha_{k m}$ different from that in (25). However, these possible improvements are beyond the scope of the present paper, where for the sake of simplicity and brevity, the somewhat standard theory described above will be used.

\section{Results}

Let us now apply the theory developed in the last section. Although this theory applies to any homogeneous material, here we consider an aluminum plate, with density $\rho=2700 \mathrm{~kg} \mathrm{~m}^{-3}$ and Lamé coefficients $\lambda=50.35 \mathrm{GPa}$ and $\mu=25.94 \mathrm{GPa}$ (Young modulus $E=69 \mathrm{GPa}$ and Poisson ratio $\nu=0.33)$. The horizontal size is $1 \times 1 \mathrm{~m}^{2}$ and the thickness is assumed to be small (say, 1-2 mm), such that the two-dimensional theory developed in the last section applies. Using the Cartesian coordinate system indicated in section 2.1 , the domain occupied by the plate is $D:-0.5$ $\mathrm{m}<x<0.5 \mathrm{~m},-0.5 \mathrm{~m}<y<0.5 \mathrm{~m}$.

As anticipated in section 1, a large number of cases have been considered and analyzed, but for the sake of brevity only the most representative ones will be presented. Concerning the healthy plate, two general classes of plates will be presented, namely:

- A homogeneous plate, with constant density and Lamé coefficients.

- An inhomogeneous plate containing an elongated through-slit or an elongated inclusion of a different material.
Concerning the emitting/sensing configurations, these will be classified into three types, namely:

- PL emitting/PL sensing.

- RSR emitting/RSR sensing.

- RSR emitting/PL sensing.

RSR actuators and sensors will always be circular. The radius of these emitting or receiving devices will always be set as $r=5 \mathrm{~mm}$, which is similar to the radius of typical piezoelectric devices. In order to obtain comparable results for the various cases that will be considered, the centers of the actuators and sensors will always be located at $2 \mathrm{~cm}$ distance from the boundary, which has been selected on purpose to be very small distance compared to the plate size and would lead to strong difficulties if standard SHM methods were used. Location of the actuators and sensors along the lines parallel to the sides of the plate will be chosen with no particular care, which will emphasize robustness.

As anticipated, the topological derivative will only be calculated in an interrogation window $D_{I}$ that must exclude a vicinity of the actuators and sensors. In the present application, the interrogation window will be a $70 \times 70 \mathrm{~cm}^{2}$ centered square, namely

$D_{I}:-70 \mathrm{~cm} \leqslant x \leqslant 70 \mathrm{~cm}, \quad-70 \mathrm{~cm} \leqslant y \leqslant 70 \mathrm{~cm}$,

which means that a $15 \mathrm{~cm}$ gap will be ignored near the boundary of the plate. The window $D_{I}$ will be the same for all considered cases, which will emphasize robustness. Since the centers of the RSR devices are $2 \mathrm{cms}$ apart from the boundary and their radii is $0.5 \mathrm{~cm}$, in order to avoid the vicinity of the actuators and sensors (where, as anticipated, the topological derivative may exhibit spurious negative peaks), the gap between the interrogation window and the boundary of the plate should be somewhat large compared to $2.5 \mathrm{~cm}$. The $15 \mathrm{~cm}$ value for the gap has been chosen after a fairly rough calibration and could be decreased, but this has not been done because the selected value is appropriate to illustrate the power of the method, which is the purpose of this article.

The defects will always be inside the interrogation window $D_{I}$ and will be circular or elliptic through-holes, with a very small size $\sim 5 \mathrm{~mm}$. As anticipated in section 2.2 , it is the negative peaks of the topological derivative that matter to localize defects. Thus, we shall consider plots of the topological derivative, which will always be rescaled as follows. The topological derivative outside $D_{I}$ will be set to zero and the positive values of the topological derivative inside $D_{I}$ will be replaced by zero. The negative values of the topological derivative inside $D_{I}$ will be rescaled with the maximum of its absolute value inside $D_{I}$. Therefore, all topological derivatives will vary in the range between 0 and -1 .

The number of available data increases as the number of actuators/sensors configurations, $M$, the number of sensors, $N$, and the number of involved frequencies, $K$, increase. However, increasing $M$ and/or $N$ requires increasing the number of devices. This is more problematic in practice than increasing $K$, which only affects the complexity of the emitting signal, according to (6). Thus, some emphasis will be put 

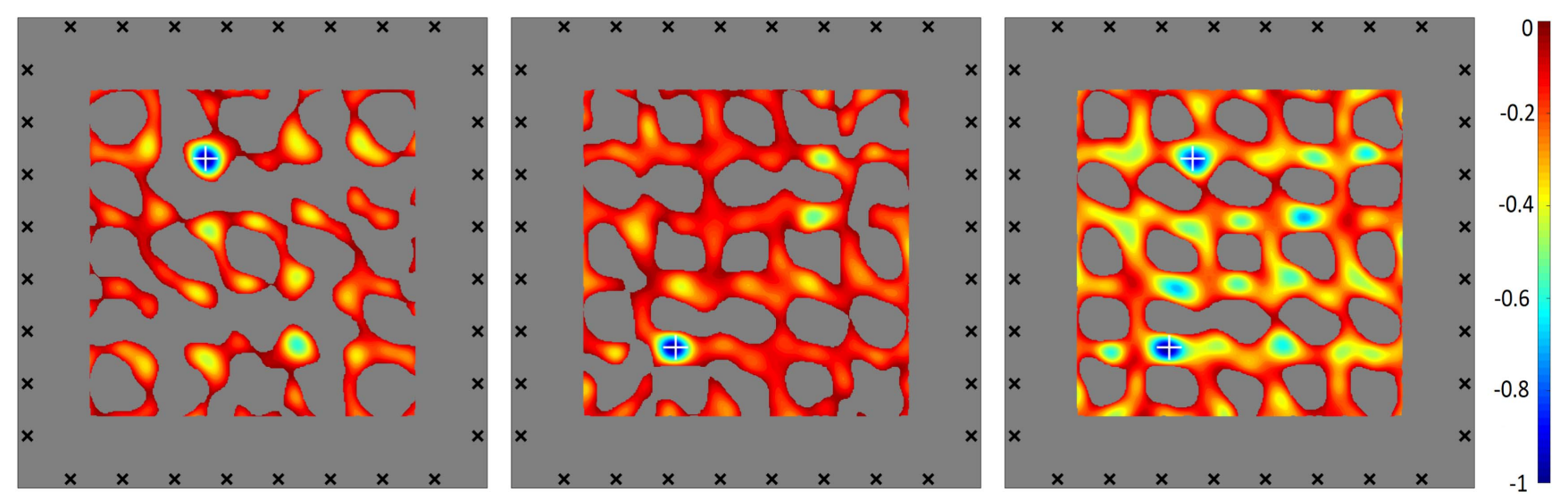

Figure 3. Color map of the topological derivative for the first emitting/receiving configuration (using $32 \mathrm{PL}$ emitting/receiving devices indicated with black $\times$ symbols), considering a circular defect of radius $2.5 \mathrm{~mm}$ (left), an elliptical defect, with major and minor axes equal to $4.84 \mathrm{~mm}$ and $1.29 \mathrm{~mm}$, parallel to the $x$ and $y$ axes, respectively (middle), and the circular-plus-elliptical defects simultaneously present (right), whose center position is indicated with white + symbols.

below in decreasing $M$ and $N$ (at the expense of increasing $K)$. On the other hand, selecting actuators/sensors that are somewhat close to each other (which makes the available data less uncorrelated) and close to the boundary of the plate favors compact designs of the experimental device and will be intended below.

Application of the method described in section 2.2 requires solving elastic problems of the type (8)-(10) for both:

- The perturbed plate, to obtain the synthetic data (mimicking experimental data).

- The healthy plate, to solve the unperturbed direct and adjoint problems, which are needed to compute the topological derivative.

The solution to these problems will be obtained using the ANSYS Mechanical FEM solver [46], with a second degree polynomial as shape function. Concerning the computational mesh, the typical size of the individual elements will be $\sim 2.5 \mathrm{~mm}$, though they will concentrate near the actuators and sensors (and elongated inhomogeneities, if present); they will also concentrate near the through-holes when calculating synthetic data. All these will give a number of degrees of freedom $\sim 0.96$ Million (corresponding to 0.48 Million nodes). An idea of the computational meshes that will be used to generate the synthetic data and to solve the unperturbed direct and adjoint problems for the case considered in figure 3-left is given in figure 2. Note that the two meshes are quite different, both quantitatively and qualitatively. The main qualitative difference between them is that nodes concentrate near the defect in the left plot (because the defect is taken into account when computing synthetic data), but not in the right plot, which corresponds to computations of the unperturbed direct and adjoint problems in the healthy plate.

In the remaining of this section, we consider several actuators/sensors types and configurations, homogeneous and inhomogeneous plates, and defects locations. In order to emphasize the robustness of the method, the forcing frequencies will be chosen as equispaced in a range that will be the same for all cases, namely

$$
10 \mathrm{kHz} \leqslant \omega / 2 \pi \leqslant 25 \mathrm{kHz} \text {. }
$$

Considering a different range, such as $10 \mathrm{kHz} \leqslant \omega \leqslant 50 \mathrm{kHz}$, gives similar results. As anticipated, PL emission is problematic. Thus, even though this type of actuator will be first considered section 3.1, the fairly good results for this case will be tested for the less problematic case of RSR actuators, with either RSR or PL sensors, in sections 3.2 and 3.3, respectively.

\subsection{PL emission and PL sensing}

To begin with, we consider a number $M$ of PL dual emitting/ receiving PL devices, which will be used to obtain (for each of the $K$ driving frequencies) $M$ emitting/receiving configurations. In each of these, signals are emitted from one of the devices (which acts as actuator and promotes a displacement parallel to the $y$ axis) and received at the remaining $N=M-1$ devices (where the whole displacement vector is measured), which act as sensors. Since two scalar data are produced at each PL sensor, the total number of available scalar data is $2 K \times M \times(M-1)$, which will be only moderately large (not larger than a few tens of thousands) in the applications below. In addition, these data are not completely uncorrelated, meaning that the effective number of data is even smaller. The number of unknowns, instead, are the values of $\rho, \lambda$, and $\mu$ at the nodes of the computational mesh, which is $\sim 3 \times 0.96=2.88$ Million unknowns, namely much larger than the number of data. This illustrates the ill-posed nature of the inverse problem. Using the topological derivative, described in section 2.2, somehow circumvents this difficulty.

3.1.1. Homogeneous plate. The first considered emitting/ receiving configuration consists of 32 evenly located (as indicated with black $\times$ symbols in figure 3) PL emitting/ receiving devices, which are fairly close to the boundary, 


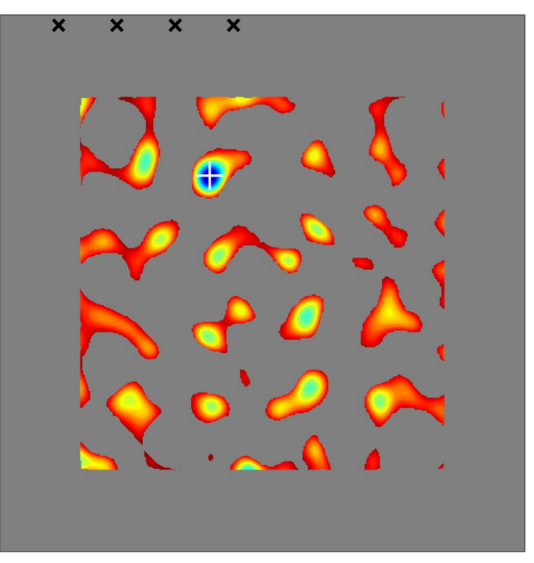

15 forcing frequencies

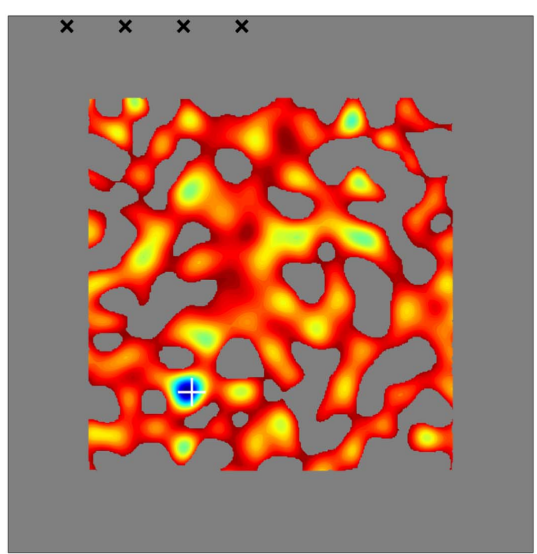

15 forcing frequencies

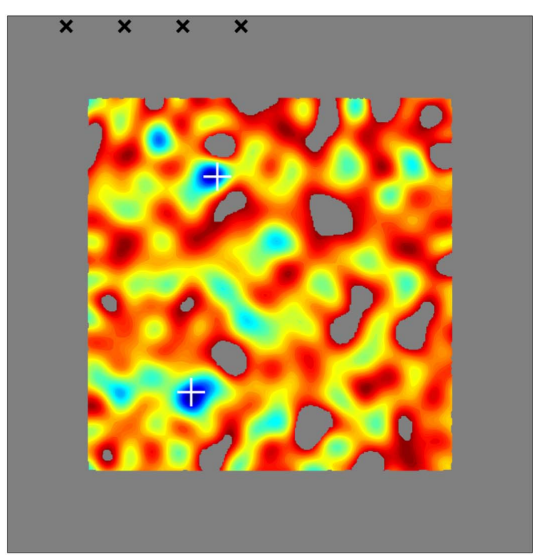

0
-0.2
-0.4
-0.6
-0.8
-1

35 forcing frequencies

Figure 4. Counterpart of figure 3 , but using only the four emitting/receiving devices located at the positions indicated with black $\times$ symbols and the indicated number of equispaced frequencies.

namely $2 \mathrm{~cm}$ apart. The devices are equispaced along the lines parallel to the sides of the boundary. Concerning the driving frequencies, we use $K=15$ equispaced values in the interval (28). For this emitting/receiving configuration, we consider three sets of through-hole defects, namely (a) a circular defect of radius $2.5 \mathrm{~mm}$, with its center at $(x, y)=(-0.1 \mathrm{~m}, 0.2 \mathrm{~m})$, (b) an elliptical defect with center at $(x, y)=(-0.15 \mathrm{~m}$, $-0.2 \mathrm{~m}$ ) and major and minor axes $4.84 \mathrm{~mm}$ and $1.29 \mathrm{~mm}$, parallel to the $x$ and $y$ axes, respectively, and (c) the circular and elliptical defects simultaneously located in the plate. Note that the two defects exhibit the same area. In figure 3 (as in all plots of the below), the actual position of the defects is indicated with white + symbols; the shapes of the defects cannot be seen in this plot due to their very small sizes. The topological derivative for these three sets of defects is as plotted in figure 3, where only negative values of the topological derivative are represented in the color map; points with positive values and points outside the interrogation window are indicated in gray. As can be seen, the negative peaks of the topological derivative locate fairly well the defects, especially in cases (a) and (b), in which only one defect is present; case (c), with two defects, is more demanding and thus the contrast of the negative peaks of the topological derivatives at the defects is less prominent. Also, the circular and elliptical defects promote comparable negative peaks in the topological derivative, which is due to the fact that both defects have been selected to exhibit the same area. If, instead, one of the through-holes exhibited a smaller area, then the (negative) peak of the topological derivative at this defect would be less prominent.

The number of emitting/receiving devices, $M=32$, was chosen in the previous test problem to ensure obtaining good results, but most likely they produce highly correlated data and can be decreased. Thus, we drastically decrease this number from 32 to 4, maintaining the same defects sizes and locations as in the previous test problem. The four considered emitting/receiving devices are located close to each other and in the upper side of the plate, as indicated with black $\times$ symbols in figure 4; in fact, they are a subset of those considered in figure 3. Concerning the number $K$ of forcing frequencies (which again are equispaced in the interval (28)), this is $K=15$, as in the previous test problem, when only one defect is present, but this number is increased to $K=35$ in the more demanding case in which both defects are present. As can be seen in figure 4, the topological derivative locates the defects fairly well using only four compactly located emitting/receiving devices. Thus, in the remaining of this subsection, we shall use only this reduced set of devices.

3.1.2. Inhomogeneous plate. The test problems considered here are much more demanding than the previous ones. Thus, they will require increasing the number of frequencies if the number of emitting/receiving devices is maintained, as anticipated above.

As a first test problem, we consider the plate with an elongated through-slit, considering two cases (see figure 5):

- A centered parallel (to the $x$ axis) slit with length $0.8 \mathrm{~m}$ and width $1.6 \mathrm{~cm}$ located along the line $y=0.17 \mathrm{~m}$, and a circular defect of radius $2.5 \mathrm{~mm}$ and center at $(x, y)=(0.1 \mathrm{~m},-0.2 \mathrm{~m})$.

- An oblique slit with length $0.7 \mathrm{~m}$ and width $1.4 \mathrm{~cm}$, located along a line with slope $40.17^{\circ}$ and centered at $(x, y)=(0.0609 \mathrm{~m}, 0.129 \mathrm{~m})$. The defect is again circular, with radius $2.5 \mathrm{~mm}$ and center at $(x, y)=(0.15 \mathrm{~m}, 0.1 \mathrm{~m})$.

For this test problem, we consider $K=30$ frequencies, equispaced in the interval (28). The topological derivatives for the parallel and oblique slits, plotted in figure 5, show that the method locates the defects reasonably well in spite of the presence of the slits. In both cases, especially for the oblique slit, the defect is not reachable by the primary emitted waves, but only by waves reflected at the boundary, which would make the application of standard SHM methods extremely problematic. In fact, through-slits are quite demanding because they totally reflect the waves, as the lateral walls do, due to the infinite jump in the acoustic impedance. Here, the slits leave a space at the borders, which allows the guided waves to pass to the other side. If, instead, the through-slits 

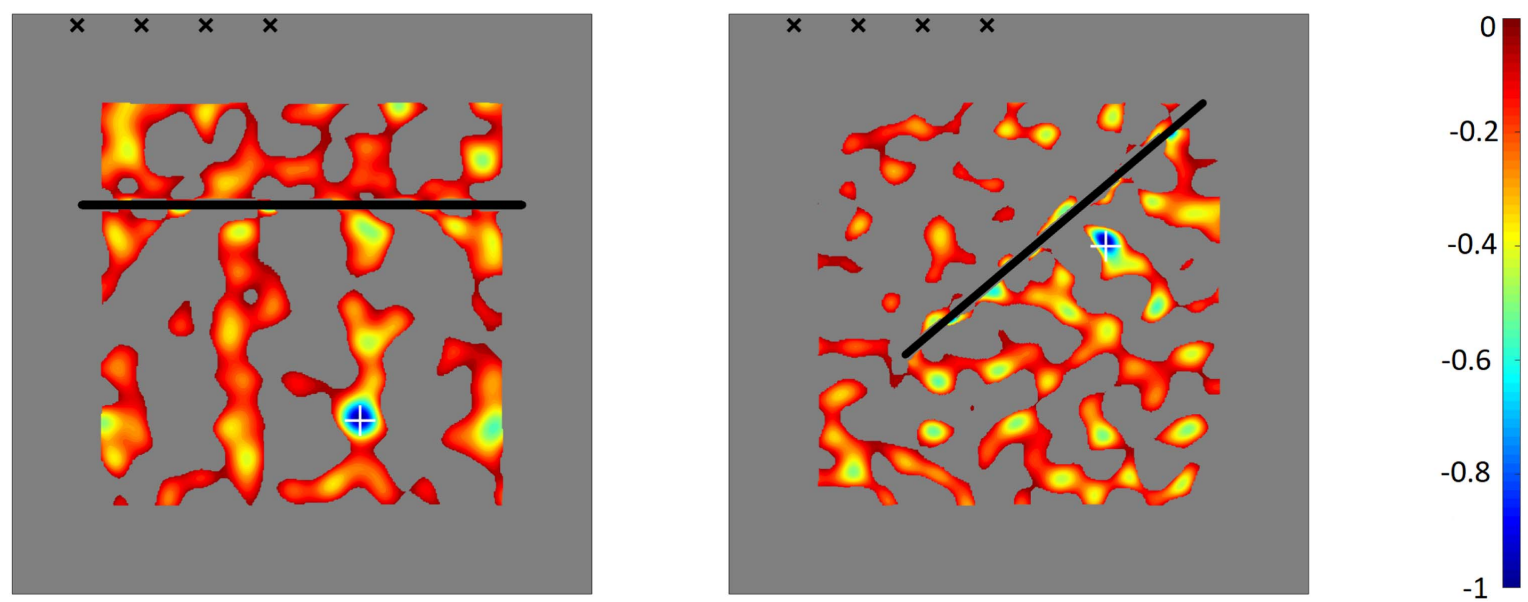

Figure 5. Color map of the topological derivative for inhomogenous plates showing the parallel (left) and oblique (right) through-slits indicated in black. As in previous figures, the emitting/receiving devices locations are indicated with black $\times$ symbolds, and the defect locations, with white + symbols.

were longer, as to completely cover a section of the plate, from side to side, it would be absolutely impossible to detect any defect beyond the slits. However, this does not happen with elongated inclusions of a different material, which are considered in the next test problem, because these inclusions allow for a part of the waves to trespass the inclusion (though they are subject to diffraction).

As a second test problem, we consider a plate with an elongated inclusion of a different material. The inclusion is parallel to the $x$ axis and transversally covers the whole plate at $y=0.16 \mathrm{~m}$ from side to side. As for the thickness and material properties of the inclusion and the nature of the material, we consider two cases:

- Titanium inclusion: titanium is a fairly dense and fairly rigid material, with density $\rho=4500 \mathrm{~kg} \mathrm{~m}^{-3}$ and Lamé coefficients $\lambda=74.07 \mathrm{GPa}$ and $\mu=41.67 \mathrm{GPa}$ (Young modulus $E=110 \mathrm{GPa}$ and Poisson ratio $\nu=0.32$ ). The thickness of the inclusion is $15 \mathrm{~mm}$. In this case, we consider $K=25$ forcing frequencies, equispaced in the interval (28).

- High-density polyethylene (HDPE) inclusion: now, both the density $\left(\rho=960 \mathrm{~kg} \mathrm{~m}^{-3}\right)$ and especially the rigidity ( $\lambda=1.777 \mathrm{GPa}$ and $\mu=0.3901 \mathrm{GPa}$, which correspond to Young modulus $E=1.1 \mathrm{GPa}$ and Poisson ratio $\nu=0.41)$ are much smaller than those for aluminum. The thickness of the inclusion is $2.5 \mathrm{~mm}$, much smaller than in the previous case since, as explained below, this is a more demanding case. Because of this, the number of forcing frequencies (again equispaced in the interval (28)) is also larger than in the previous case, namely $K=60$.

In both cases, the defect that is to be detected is circular, with radius $2.5 \mathrm{~mm}$ and centered at $(x, y)=(0.1 \mathrm{~m},-0.2 \mathrm{~m})$.

The topological derivatives for these two cases are as plotted in figure 6. As can be seen in this figure, the most demanding case is that of HDPE, which involves a smaller thickness of the inclusion and requires a larger number of frequencies. The reason for being so demanding is that both the density and the Lamé coefficients are much smaller than that of the aluminum plate. This makes this case closer to the elongated through-slit, which as anticipated is the most demanding inhomogeneity.

In any event, it is remarkable how the method is able to capture defects that are beyond the elongated through-slits covering a wide portion of the transversal section of the plate and elongated inclusions of different material covering the whole section of the plate, from side to side.

\subsection{RSR emission and RSR sensing}

Let us now consider dual emitting/receiving devices of the RSR type. Since RSR sensors only capture one scalar quantity (instead of two), a larger (than in the PL case) number of devices and a larger number of frequencies are needed in the present case. Thus, we consider eight RSR devices of radius $5 \mathrm{~mm}$ and centers located at $2 \mathrm{~cm}$ from the $y=0.5 \mathrm{~m}$ side of the plate, equispaced in the $x$ coordinate (along the horizontal line $y=0.48 \mathrm{~m}$ ).

For the homogeneous plate, we consider the same defects configurations as in figure 4 and an appropriate number of forcing frequencies. The obtained topological derivatives are given in figure 7. As can be seen in this figure, the topological derivative identifies the defects reasonably well, especially when only one defect is present, though the number of required frequencies is larger than in the case considered in figure 4 , as expected. Note that two additional blue regions appear in the case of two defects. However, the topological derivative peaks at these additional regions are less intense than near the actual position of the defects (which means that the topological derivative locates well the defects, as stated).

Concerning the case of the inhomogeneous plates considered in section 3.1.2, with the same elongated slits or inclusions and defects as in figures 5 and 6 , the topological derivatives for the present actuators/sensors configuration are given in figure 8 . As can be seen in this figure, the topological derivative locates the defects quite well using an appropriate number of forcing frequencies (again, equispaced in the interval (28)), namely 60 and 40 frequencies for the parallel 


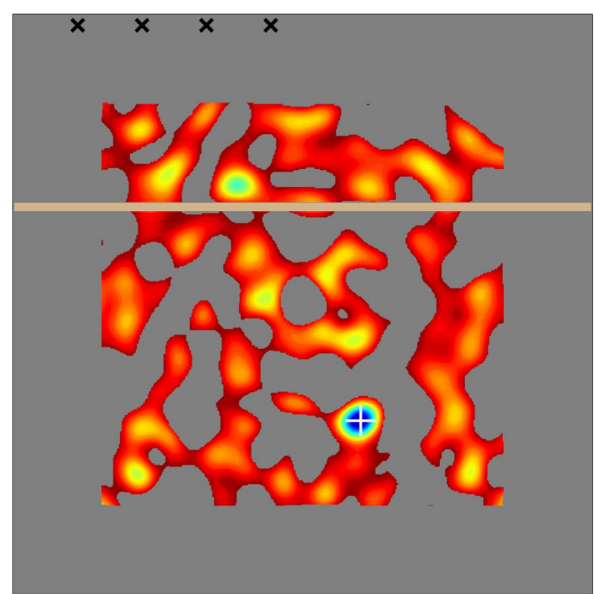

25 forcing frequencies
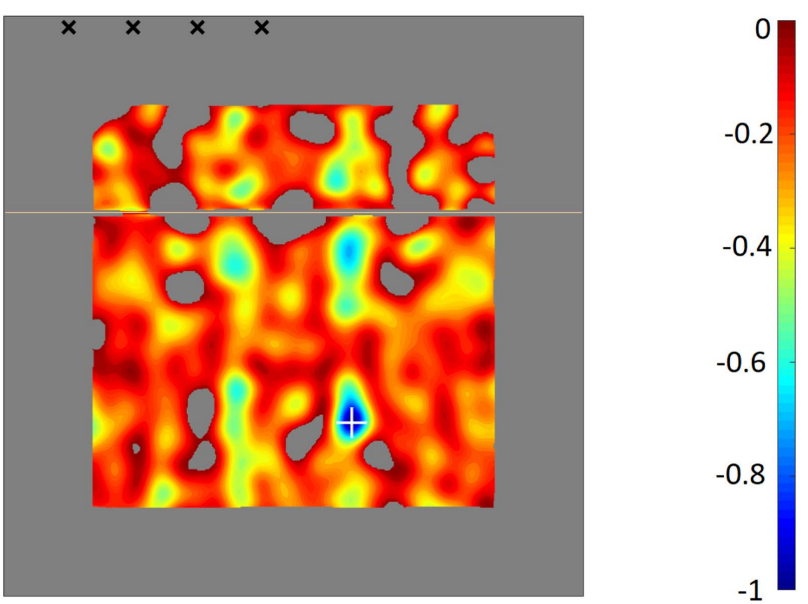

60 forcing frequencies

Figure 6. Color map of the topological derivative for inhomogenous plates showing horizontal inclusions (indicated in light brown) of titanium (left) and HDPE (right). As in previous figures, the emitting/receiving devices locations are indicated with black $\times$ symbols, and the defect locations, with white + symbols.

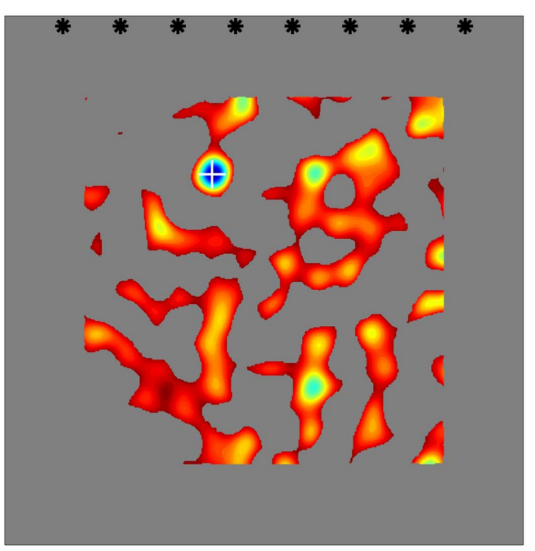

40 forcing frequencies

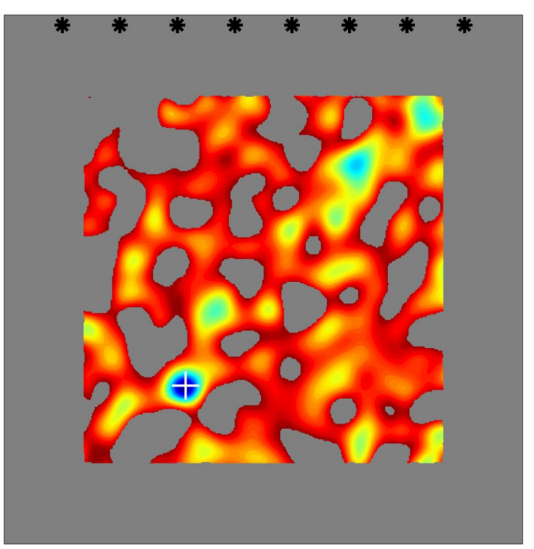

40 forcing frequencies
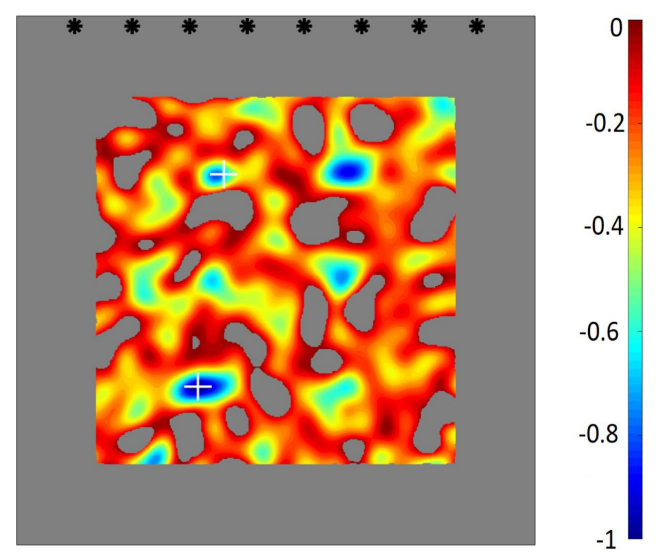

60 forcing frequencies

Figure 7. Counterpart of figure 4, using the eight RSR emitting/receiving devices located at the positions indicated with black asterisks and the indicated number of equispaced frequencies in the interval (28).

and oblique through-slits, respectively, and 40 and 60 frequencies for the titanium and HDPE inclusions, respectively. Note that the number of frequencies is larger for the HDPE inclusion than for the titanium inclusion because, as already mentioned, this material is much more demanding in connection with inclusions.

Summarizing, the results obtained for this new type of dual RSR actuators/RSR sensors are consistent with those obtained in section 3.1.2 for dual PL actuators/PL sensors. In particular, as anticipated, since RSR sensors collect a smaller amount of data than PL sensors, the numbers of required devices and frequencies are larger for the present case.

\subsection{RSR emission and PL sensing}

Let us now consider the case of RSR actuators and PL sensors. Since, as anticipated, PL sensors collect a larger amount of data (namely, twice as much) than RSR sensors, the present case is less demanding than that considered in the last subsection. This means that we may decrease either the number of actuators or the number of sensors, which must be located in different positions (but again $2 \mathrm{~cm}$ apart from the boundary) because they are of a different type among each other. We select the same four RSR actuators as in section 3.2 and four PL sensors aligned with the actuators in the line $y=0.48 \mathrm{~m}$ and equispaced along the $x$ coordinate in the right part of this line. Actuators and sensors are indicated with black asterisks and black $\times$ symbols, respectively, in figures 9 and 10, which show the topological derivatives for the homogeneous and inhomogeneous plates, respectively, considering the same defects (and slits or inclusions) as in figures 7 and 8, respectively. As can be seen, the topological derivative locates the defects quite well in all considered cases.

Summarizing the results obtained in this subsection, RSR emision and PL sensing gives results similar to PL emision and PL sensing because, as anticipated, these two methods 


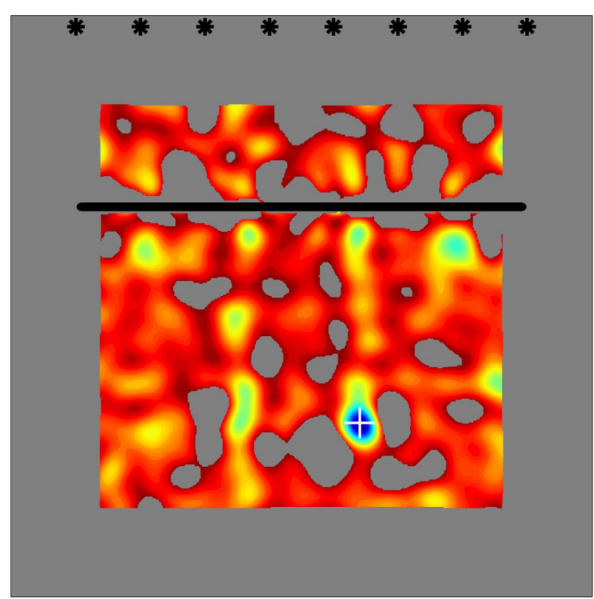

60 forcing frequencies

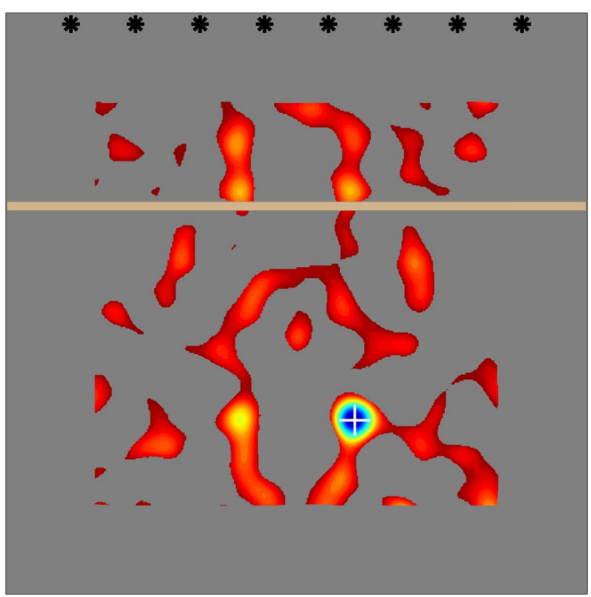

40 forcing frequencies
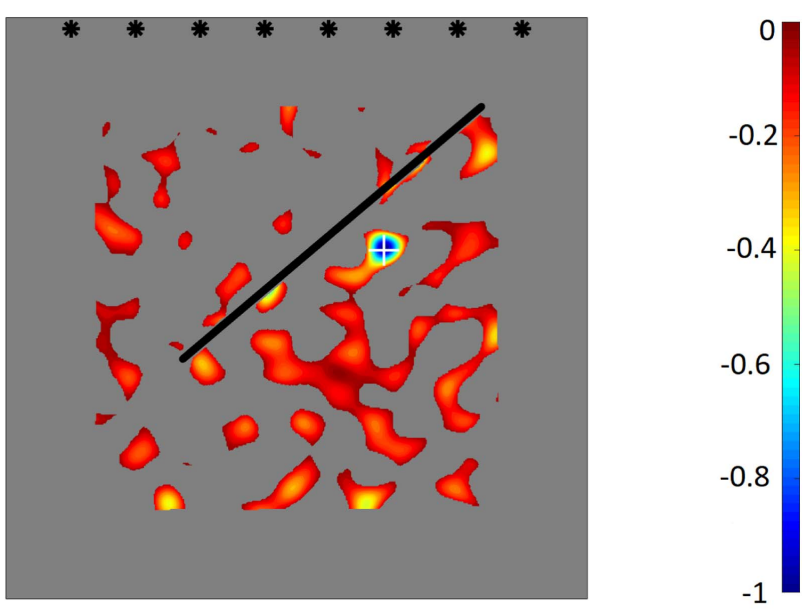

40 forcing frequencies
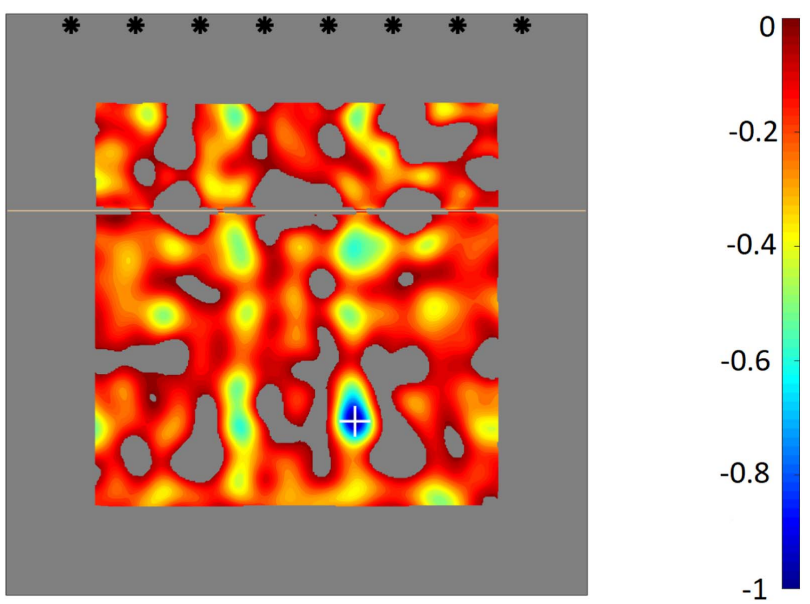

60 forcing frequencies

Figure 8. Color map of the topological derivative for inhomogenous plates. Top plots: parallel (left) and oblique (right) through-slits indicated in black. Bottom plots: titanium (left) and HDPE (right) inclusions. As in figure 7, the emitting/receiving devices locations are indicated with black asterisks, and the defect locations, with white + symbols.

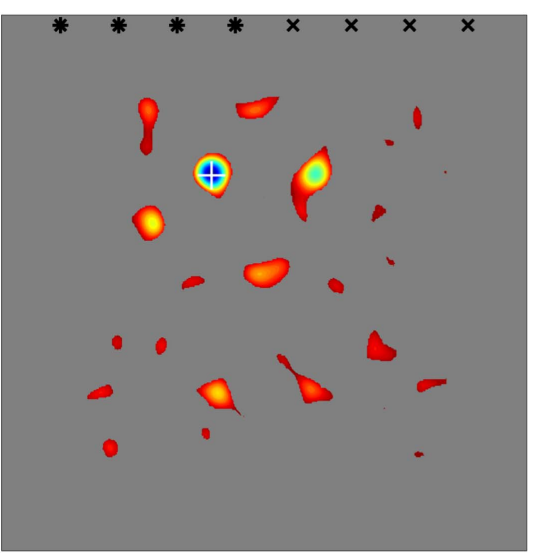

30 forcing frequencies

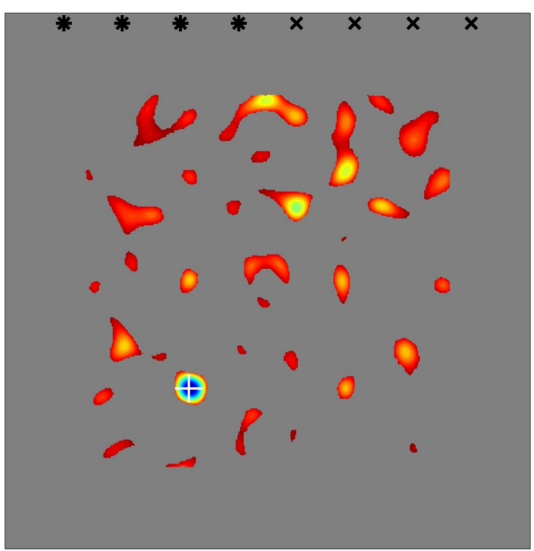

30 forcing frequencies

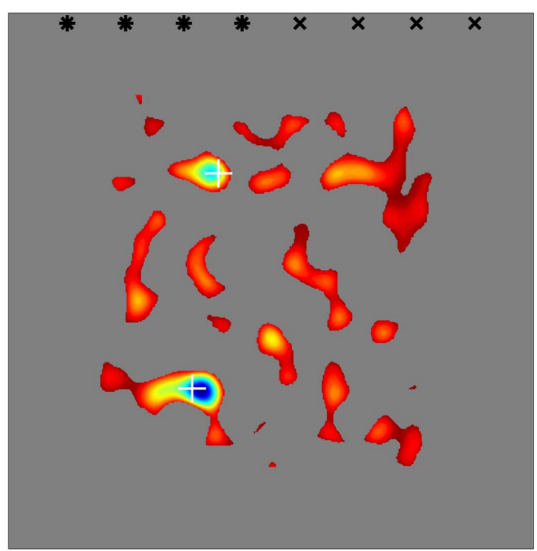

0
-0.2
-0.4
-0.6
-0.8
-1

35 forcing frequencies

Figure 9. Counterpart of figure 7 for RSR emission and PL sensing. 


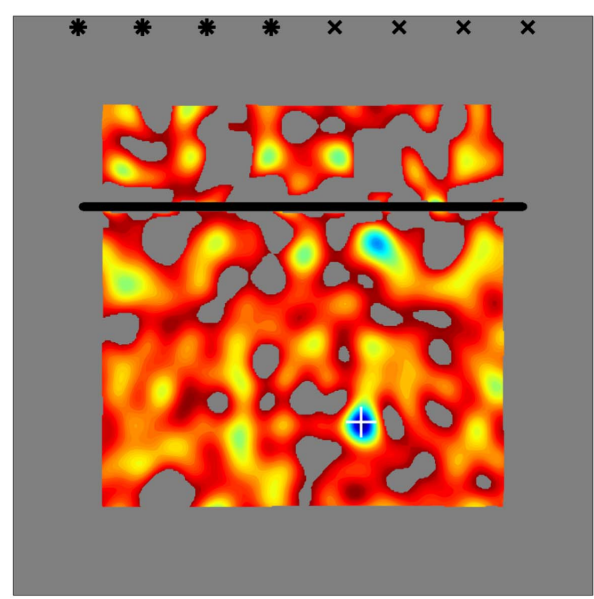

40 forcing frequencies

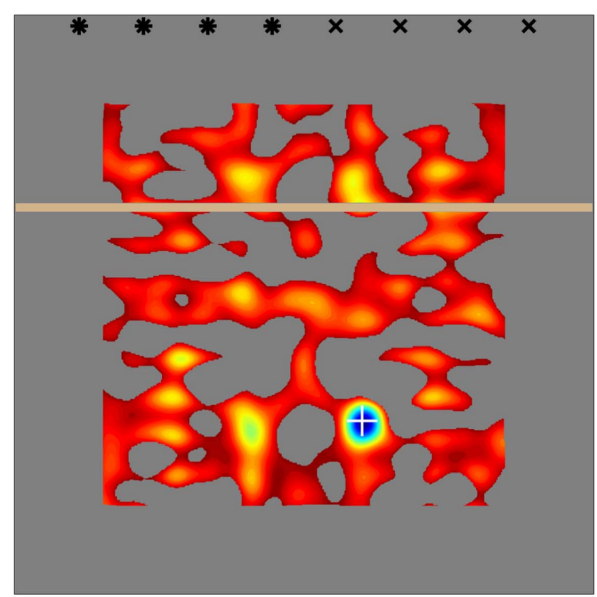

30 forcing frequencies
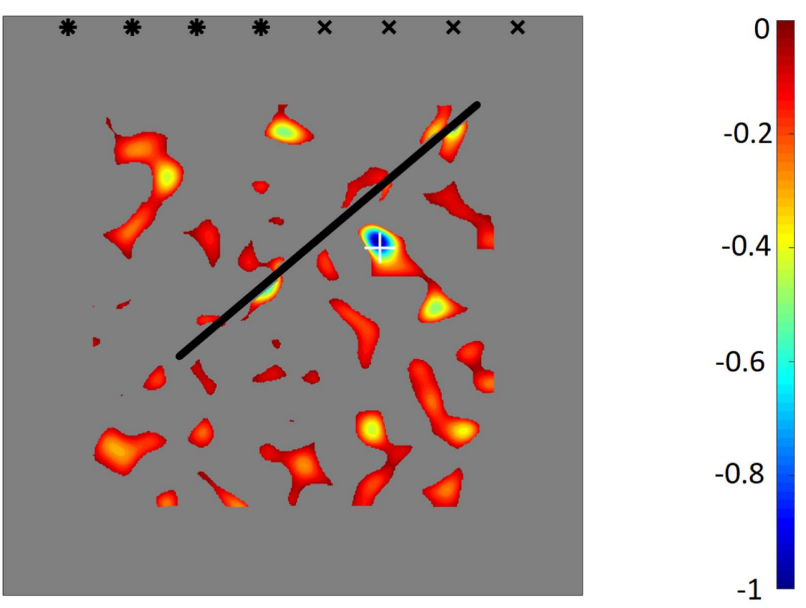

\section{0 forcing frequencies}
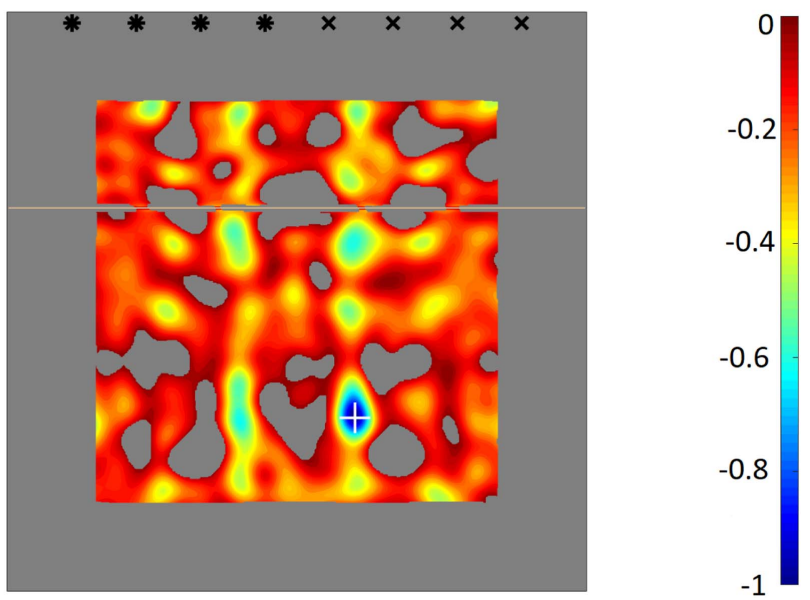

60 forcing frequencies

Figure 10. Counterpart of figure 8 for RSR emission and PL sensing.

provide comparable number of data (which mainly depends on sensing).

\section{Conclusions}

A method based on the multi-frequency topological derivative has been developed that is intended as an advantageous alternative to standard guided-waves-based SHM methods. The main advantage of this new method is that it uses the whole physics of the problem, instead of only a part of the physics, as standard methods do. As a proof of the concept, the method has been applied using synthetic data, instead of plain experimental data, and considering only symmetric guided waves, produced by in-plane excitation of two types, either PL or RSR excitation. Likewise, data is collected at some sensors, which are either of the PL or RSR type. The emitting and sensing devices have been located very close to the boundary of the plate, which is a very demanding situation for standard SHM methods. The 'healthy' plate can either be homogeneous or inhomogenous, showing either elongated through-slits or elongated inclusions of a different material, which is again too demanding for standard SHM methods. In all cases, the new method gave good results using a very limited number of emitting and sensing devices.

A number of issues have been left apart in this first presentation of the method:

- Using actual experimental data instead of synthetic data. This obviously requires having these experimental data available, and also to:

1. Clean the (necessarily noisy) experimental data.

2. Calibrate the numerical modeling of the healthy plate elastodynamics, including the piezoelectric and laser Doppler devices.

Thus, this issue involves additional, fairly strong difficulties that are well beyond the scope of this paper, which aims at just presenting the proposed methodology as a proof of the concept demonstration. Using actual experimental data is obviously the final end, but it has been not addressed for the reasons given. 
- Considering general Lamb waves instead of just symmetric waves. This can be done with the appropriate ANSYS solver, though the computational cost would be higher.

- Reducing the computational cost of solving the unperturbed direct and adjoint problems, which could be done via an appropriate reduced order model [49]. It must be noted here that these two problems are defined in terms of the healthy plates and are linear in connection with the forcing and sensing intensities. Thus, they may be written in terms of canonical problems that can be solved offline beforehand. Using the solutions of these canonical problems, the computational cost of the online step to compute the topological derivative is fairly small.

However, these issues are beyond the scope of this paper, and left as the object of future research.

\section{Acknowledgments}

The work by AM, JMP, and JMV was supported by the Spanish Ministry of Economy and Competitiveness under the grant TRA2016-75075-R. We are also indebted to two anonymous referees for very useful comments on an earlier version of the paper.

\section{ORCID iDs}

J M Perales (ib https://orcid.org/0000-0001-9873-8115

J M Vega (1) https://orcid.org/0000-0002-4307-9623

\section{References}

[1] Atkin R J and Fox N 2005 An Introduction to the Theory of Elasticity (Mineola: Dover)

[2] Balageas D, Fritzen C P and Güemes A 2010 Structural Health Monitoring (New York: Wiley)

[3] Larrabide I, Novotny A A, Feijóo R A and Taroco E 2005 A medical image enhancement algorithm based on topological derivative and anisotropic diffusion Proc. 26th Iberian Latin-American Congress on Computational Methods in Engineering-CILAMCE pp 1-14

[4] Meju M A 2002 Geoelectromagnetic exploration for natural resources: models, case studies and challenges Surv. Geophys. 23 133-205

[5] Wunsch C 1996 The Ocean Circulation Inverse Problem (Cambridge: Cambridge University Press)

[6] Scott W R, Schroeder C T, Martin J S and Larson G D 2001 Use of elastic waves for the detection of buried land mines 2001 Int. Geoscience and Remote Sensing Symposium, 2001. IGARSS'01 vol 3 (IEEE) pp 1116-8

[7] Lin B and Giurgiutiu V 2006 Modeling and testing of PZT and PVDF piezoelectric wafer active sensors Smart Mater. Struct. 15 1085-93

[8] Giurgiutiu V 2007 Structural Health Monitoring: With Piezoelectric Wafer Active Sensors (New York: Academic)

[9] Royer D, Noroy M H and Fink M 1994 Optical generation and detection of elastic waves in solids $J$. Physique IV 4 673-83
[10] Staszewski W J, Lee B C, Mallet L and Scarpa F 2004 Structural health monitoring using scanning laser vibrometry: I. Lamb wave sensing Smart Mater. Struct. 13 251-60

[11] Leong W H, Staszewski W J, Lee B C and Scarpa F 2007 Structural health monitoring using scanning laser vibrometry: III. Lamb waves for fatigue crack detection Smart Mater. Struct. 14 1387-95

[12] Staszewski W J, Lee B C and Traynor R 2007 Fatigue crack detection in metallic structures with Lamb waves and 3D laser vibrometry Smart Mater. Struct. 18 727-39

[13] Yu L and Tian Z 2013 Lamb wave structural health monitoring using a hybrid pzt-laser vibrometer approach Struct. Health Monit. 12 469-83

[14] Lee J R, Chong S Y, Sunuwar N and Park C Y 2013 Repeat scanning technology for laser ultrasonic propagation imaging Meas. Sci. Technol. 24085201

[15] Luo M, Li W, Wang J, Wang N, Chen X and Song G 2018 Development of a novel guided wave generation system using a giant magnetostrictive actuator for nondestructive evaluation Sensors 18779

[16] Hansen P C 2010 Discrete Inverse Problems: Insight and Algorithms (Philadelphia: Society for Industrial and Applied Mathematics)

[17] Kac M 1966 Can one hear the shape of a drum? The Am. Math. Mon. 73 1-23

[18] Protter M H 1987 Can one hear the shape of a drum? Revisited SIAM Rev. 29 185-97

[19] Kabanikhin S I 2008 Definitions and examples of inverse and ill-posed problems J. Inv. Ill-Posed Problems 16 317-57

[20] Tarantola A 2005 Inverse Problem Theory and Methods for Model Parameter Estimation (Philadelphia: Society for Industrial and Applied Mathematics)

[21] Lee B C and Staszewski W J 2003 Modelling of Lamb waves for damage detection in metallic structures: II. Wave interactions with damage Smart Mater. Struct. 12 815-24

[22] Lee B C and Staszewski W J 2007 Lamb wave propagation modelling for damage detection: I. Two-dimensional analysis Smart Mater. Struct. 16 249-59

[23] Lee B C and Staszewski W J 2007 Sensor location studies for damage detection with Lamb waves Smart Mater. Struct. 16 399-408

[24] Belanger P, Cawley P and Francesco Simonetti F 2010 Guided wave diffraction tomography within the Born approximation IEEE Trans. Ultrason. Ferroelectr. Freq. Control 57 1405-18

[25] Huthwaite P and Simonetti F 2013 High-resolution guided wave tomography Wave Motion 50 979-93

[26] Chan E, Rose L R F and Wang C H 2015 An extended diffraction tomography method for quantifying structural damage using numerical Green's functions Ultrasonics 59 1-13

[27] Rao J, Ratassepp M and Fan Z 2016 Guided wave tomography based on full-waveform inversion IEEE Trans. Ultrason. Ferroelectr. Freq. Control 63 737-45

[28] Li D, Yu H, Xu F, Liu D P, Zhang J Q and Ta D A 2017 An $l_{p}$-norm approach to robust probabilistic inspection of platelike structure defects with guided waves Smart Mater. Struct. 26104003

[29] Hettlich F and Rundell W 1996 Iterative methods for the reconstruction of an inverse potential problem Inverse Problems 12 251-66

[30] Sokolowski J and Zolesio J P 1992 Introduction to Shape Optimization (Berlin: Springer)

[31] Higuera M, Perales J M, Rapún M L and Vega J M 2016 Combining active thermographics and topological derivative Proc. 8th European Workshop On Structural Health Monitoring

[32] Feijoo G R 2004 A new method in inverse scattering based on the topological derivative Inverse Problems 20 1819-40 
[33] Ammari H, Garnier J, Jugnon V and Kang H 2012 Stability and resolution analysis for a topological derivative based imaging functional SIAM J. Control Optim. 50 48-76

[34] Guzina B B and Bonnet M 2004 Topological derivative for the inverse scattering of elastic waves Q. J. Mech. Appl. Math. 57 161-79

[35] Bonnet M and Guzina B B 2004 Sounding of finite solid bodies by way of topological derivative Int. J. Numer. Methods Eng. 61 2344-73

[36] Guzina B B and Chikichev I 2007 From imaging to material identification: a generalized concept of topological sensitivity J. Mech. Phys. Solids. 55 245-79

[37] Ammari H, Bretin E, Garnier J, Jing W, Kang H and Wahab A 2013 Localization, stability, and resolution of topological derivative based imaging functionals in elasticity SIAM $J$. Imaging Sci. 50 48-76

[38] Serrano R 2014 Detección de grietas en placas con técnicas multifrecuencia Master Thesis Universidad Politécnica de Madrid, Spain

[39] Funes J F, Perales J M, Rapún M L and Vega J M 2016 Defect detection from multi-frequency limited data via topological sensitivity J. Math. Imaging Vis. 55 19-35

[40] Le Clainche S and Vega J M 2017 Higher order dynamic mode decomposition SIAM J. Appl. Dyn. Syst. 16 882-925

[41] Le Clainche S, Vega J M and Soria J 2017 Higher order dynamic mode decomposition for noisy experimental data: the flow structure of a zero-net-mass-flux jet newblock Exp. Therm. Fluid Sci. 88 336-53

[42] Le Clainche S, Sastre F, Vega J M and Velázquez A 2017 Higher order dynamic mode decomposition applied to postproces a limited amount of PIV data AIAA 2017-3304. 47th AIAA Fluid Dynamics Conference, AIAA Aviation Forum (Denver, CO) (https://doi.org/10.2514/ 6.2017-3304)

[43] Le Clainche S, Lorente L and Vega J M 2018 Wind prediction upstream wind turbines from a Lidar database Energies 11543

[44] Moreno R, Le Clainche S, Vega J M and Taylor P 2017 Flight flutter testing using higher order dynamic mode decomposition Int. Forum on Aeroelascity and Structural Dynamics (IFASD 2017)

[45] Lamb H 1917 On waves in an elastic plate Proc. R. Soc. A 93 $114-28$

[46] ANSYS Mechanical https://ansys.com/products/structures

[47] Bellis C and Bonnet M 2010 A fem-based topological sensitivity approach for fast qualitative identification of buried cavities from elastodynamic overdetermined boundary data Int. J. Solids Struct. 47 1221-42

[48] Oberai A A, Gokhale N H and Feijoo G R 2003 Solution of inverse problems in elasticity imaging using the adjoint method Inverse Problems 19 297-313

[49] Koutsovasilis P 2009 Model order reduction in structural mechanics PhD Thesis University of Dresden 